\title{
SEARChing For FAVOURABLe PoWder BED FUSION SETTINGS IN SinTERING OF MARAGING STEEL MS1
}

\author{
Martin Nozar, Ivana Zetková \& Ondřej Hronek
}
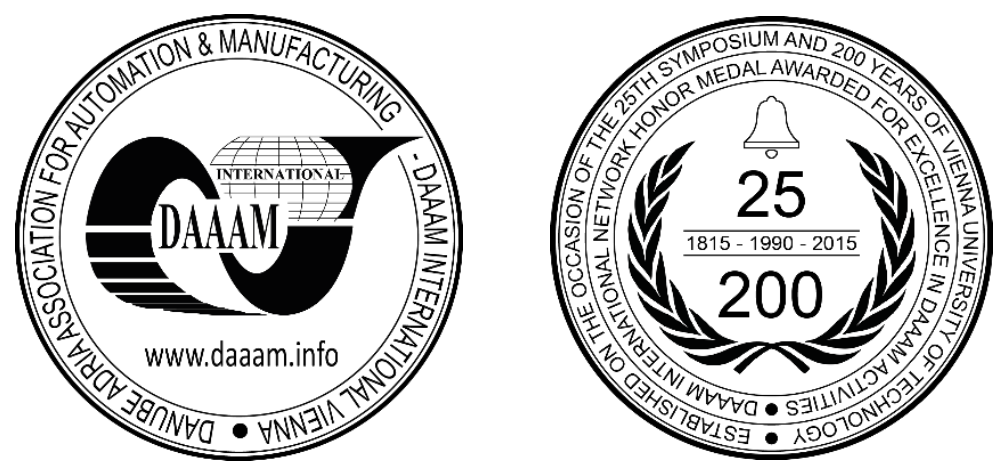

This Publication has to be referred as: Nozar, M[artin]; Zetkova, I[vana] \& Hronek, O[ndrej] (2018). Searching for Favourable Powder Bed Fusion Settings in Sintering of Maraging Steel MS1, Proceedings of the 29th DAAAM International Symposium, pp.0777-0785, B. Katalinic (Ed.), Published by DAAAM International, ISBN 978-3-90273420-4, ISSN 1726-9679, Vienna, Austria

DOI: $10.2507 / 29$ th.daaam.proceedings.113

\begin{abstract}
Additive manufacturing (AM), also known as 3D printing, enables the creation of complex shapes and structures virtually unattainable by classical production processes. This modern manufacturing technology includes metal additive technologies that are use metal powder for producing prototypes or parts with extraordinarily complicated internal or external structures. One of these technologies is direct metal laser sintering (DMLS), which is based on the powder bed fusion principle. This article deals with this principle and explains its mechanism and specifics, which are decisive for sintering every single layer and the parameters of all the final parts. The sintering process usually uses default parameters that are universal for a wide range of structures - from tiny pieces with thin walls to large and bulky parts. In such cases, parameters optimized for specific structures and situations are more appropriate. Such optimizations could enable the sintering of parts with better surface quality, with less deformations, more subtle support structures and even faster speeds. This article describes the available setting options, the processes happening inside powder bed fusion as well as an experiment focused on searching for suitable combinations of process parameters conducted on the EOS M290 machine using a martensite-hardenable steel called EOS MaragingSteel MS1.
\end{abstract}

Keywords: additive manufacturing; powder bed fusion; process parameters; operating window

\section{Introduction}

Metal additive technologies have witnessed intensive development in recent years and have found their applications not only in industrial and prototype manufacturing. However, these technologies still have a number of unique specifics and limitations, which are connected above all with the basic mechanism of powder melting and its processing requirements. The user of an AM machine has to fully understand this mechanism to correctly set up processing parameters and take advantage of the possibilities offered by some of these technologies.

This article is focused on Direct Metal Laser Sintering (DMLS), which was used in the experiment described below. DMLS belongs to the category of powder bed fusion methods, which uses a laser as the power source to sinter powdered material. The production of satisfactory parts using this powder bed fusion process requires optimum process parameters. Powder bed temperature, laser power, scan speed and scan spacing must be balanced to provide the best trade-off between dimensional accuracy, surface finish, build rate and mechanical properties. 
High-laser-power combined with low-part-bed-temperatures result in an increased tendency for non-uniform shrinkage and the build-up of residual stresses; leading to curling of parts [1]. In the worst scenario, too low total energy input will leave the part weak and only partially sintered.

Too high energy levels will result in part growth by sintering of excess surrounding powder to the part and/or degradation of the surrounding powder to the point where it cannot be easily recycled. Therefore, it is necessary to use default and non-optimal but universal parameters all the time or try to find combinations of processing parameters that provide the best result in cases of particular structures. For example, there is a big difference between sintering thin wall parts and large volumes - proper powder fusion requires specific energy input but thicker structures react to this energy in different way from thin ones.

The energy in the form of laser heat has to be delivered to the powder bed, kept for an exact length of time and then it is conducted out of the melt pool and into the surrounding solid metal. And it is in this respect that thick and thin structures are very different. In the case of unsuccessful heat dissipation, this heat usually causes thermal gradients in the sintered pieces, deforms the created layers or whole parts, and causes other unwanted processes.

However, it is possible to prevent these undesirable processes by setting more suitable parameters for given conditions. To be able to do so, it is necessary to be familiar with the powder fusion mechanism, understand the available settings and options of a particular AM machine, and make some tests to verify the results of these settings. After that we can quite easily speed up the sintering process, effectively eliminate unnecessary thermal gradients and, in doing so, also reduce the volume of necessary support structures and heat-induced deformations.

\section{DMLS technology principle and its parameters}

Direct Metal Laser Sintering [9] is one of the major additive methods used to manufacture metal components. In a nutshell, this method operates as follows: A 3D model in "stl" format is redesigned as required (above all, any surface errors and inappropriate shapes are eliminated), support structures are generated, road paths are calculated and then the model is digitally cut into discrete slices.

These slices are sent to the DMLS machine, which recombines them in a layer-by-layer sequence. The machine selectively scans the surface of the metal powder bed with a fibre laser, effectively creating a thin, planar slice of solid part geometry. Once the sintering of the layer is complete, a new specific increment of metal powder is deposited and the sintering of the next layer commences. This cycle is repeated until the build is complete.

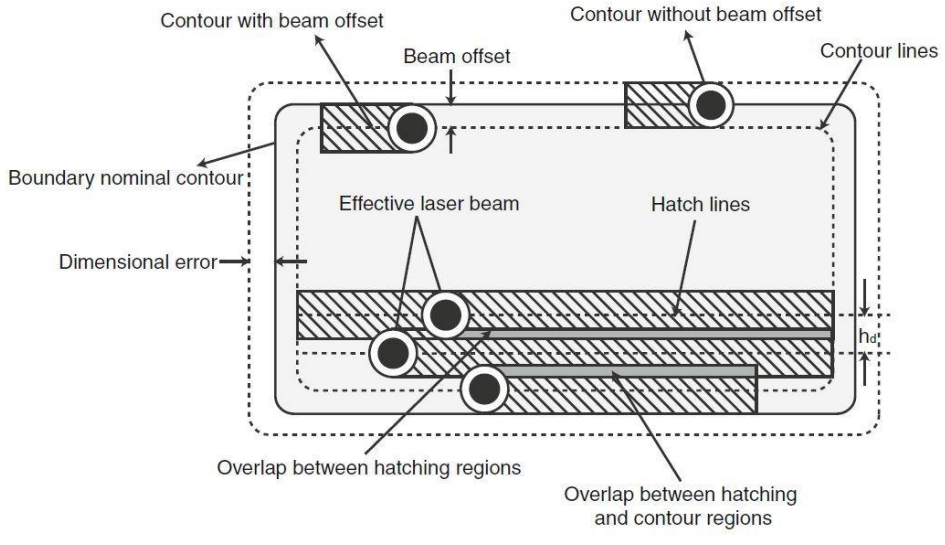

Fig. 1. Exposure strategies [4]

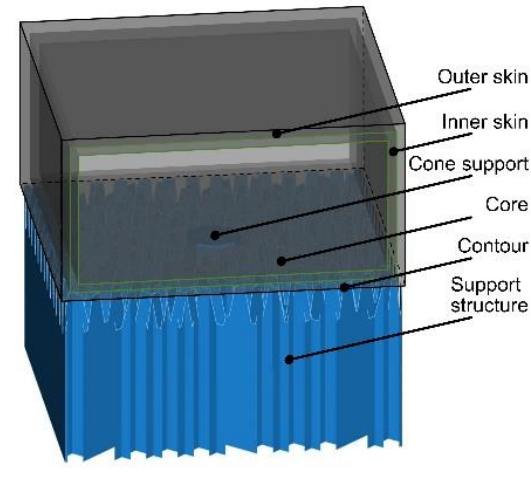

Fig. 2. Exposure types

During the exposure of a layer for a part, the laser beam moves over the surface of the bed of powder. Hatching of the enclosed areas of the layer within the contour produces a solidified layer. During this process, the laser beam moves within the cross-section of the part along parallel paths (hatch lines) with the constant application of energy [8]. (see Fig. 1). This hatch is further broken into subgroups of 'Inskin' (exposed areas are over and under it), 'Upskin' (no exposed area over it) and 'Downskin' (no exposed area under it) hatch. Inskin hatching, in the form of stripes, is used for the majority of the surface.

Such stripes on every layer can be rotated (usually by $67^{\circ}$ or $90^{\circ}$ ) prior to the next exposure. For cases in which a part is, for instance, angled relative to the building platform the Upskin and Downskin hatching is used near the surface to achieve the best possible finish. The exposure type designed for creating an accurate surface is called 'Contour' and typically consists of an inner and outer contour (see Fig. 2). A special feature allowing a different exposure in the inner part called the 'Core', can be exposed in a chessboard or hatching-like method [8]. These exposure types along with other parameters predetermine the properties of the layers to be exposed and result in complete parts. All these strongly interdependent parameters define how much energy is applied and how fast. 
In general, critical process parameters can be lumped into four categories [1]:

- Laser-related parameters (laser power, spot size, pulse duration, pulse frequency, etc.)

- Scan-related parameters (scan speed, scan spacing, and scan pattern)

- Powder-related parameters (particle shape, size and distribution, powder bed density, layer thickness, material properties, etc.)

- Temperature-related parameters (powder bed temperature, powder feeder temperature, temperature uniformity, etc.)

\section{Powder bed fusion mechanism}

\subsection{Applied energy density}

The process parameters govern the way in which laser energy is transferred into the powder bed, as described in [1]. The Melt pool formation and characteristics are fundamentally determined by the total amount of applied energy, which is absorbed by the powder bed as the laser beam passes. As a rule, the longer the laser dwells in a particular location, the deeper the fusion depth and the larger the melt pool diameter. Operating at lower laser powers requires the use of lower scan speed in order to ensure proper particle fusion. Above all, the melt pool size is highly dependent upon the setting of laser power, scan speed, spot size and bed temperature. Scan spacing should be selected to ensure a sufficient degree of melt pool overlap between adjacent lines of used material to ensure robust mechanical properties.

Both the melt pool size and melt pool depth are a function of absorbed energy density [5]. This energy density $E_{D}$ (also known as the Andrew number) can be calculated using following equation (1):

$$
E_{D}\left[\frac{J}{m^{3}}\right] \equiv \frac{\text { Laser power }[W]}{\text { Hatch distance }[\mathrm{mm}] . \text { Layer thickness }[\mathrm{mm}] \text {.Scan speed }[\mathrm{mm} / \mathrm{s}]}
$$

Although this equation does not include factors such as powder absorptivity, heat of fusion, heat accumulation (energy input into the powder bed could be faster than its cooling), laser spot size and other relevant parameters, it provides the simplest analytical approach for optimizing machine performance for a material. For a given material, laser spot size and machine configuration, a series of experiments can be run to determine the minimum applied energy necessary to achieve adequate material fusion for the desired material properties. Subsequently, build speed can be maximized by utilizing the fastest combination of laser power, scan rate and scan spacing with respect to particular circumstances (material, sintered parts structures etc.).

\subsection{Efficient powder bed fusion}

The desired settings of powder bed fusion mean the right combinations of speed and power that generates a stable melt pool of the optimum size. Such combinations are to be found in the operating window as is shown on the P-V diagram in Fig. 3. It is where the laser energy is being efficiently absorbed by the powder, creating a melt pool of sufficient depth to fuse strongly with the layer below whilst avoiding excessive re-melting.

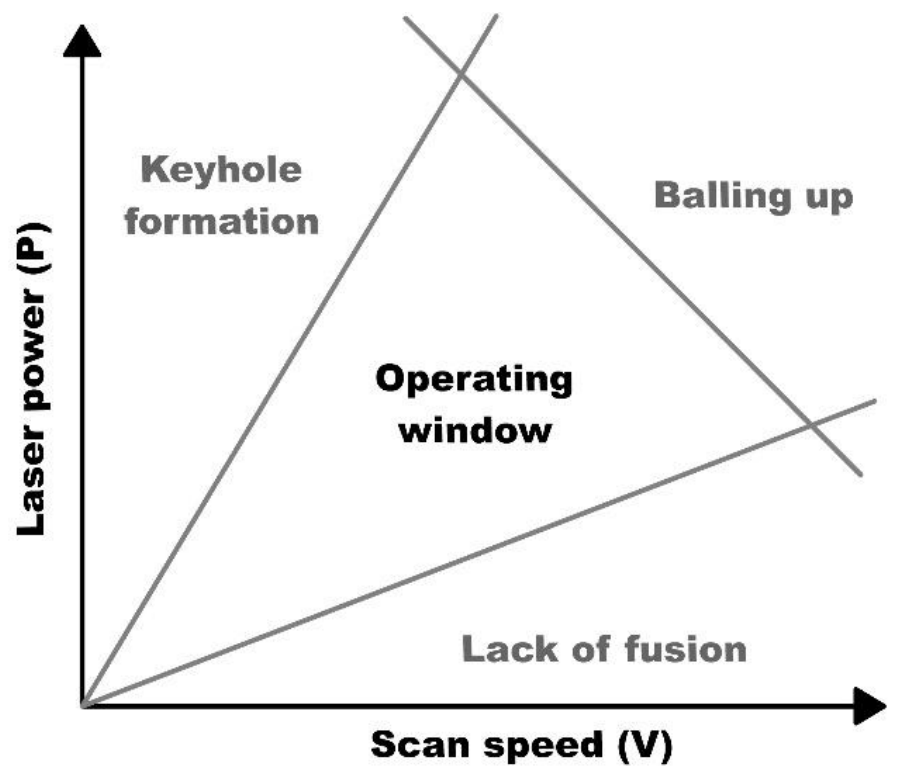

Fig. 3. Laser power vs scan speed graph [2] 
As explained in [2], in this processing zone, the laser recoil pressure creates a shallow cavity. The laser heats the front face of this cavity as it moves, creating a metal vapour plume that is ejected normal to the surface - i.e. upwards and backwards. The shallow cavity does not allow for internal reflections, so no additional melting occurs. Heat energy is conducted into the melt pool, which experiences a degree of turbulent flow due to the high temperature gradients within it and the surface tension. This flow will result in some matter being ejected in the form of weld spatter.

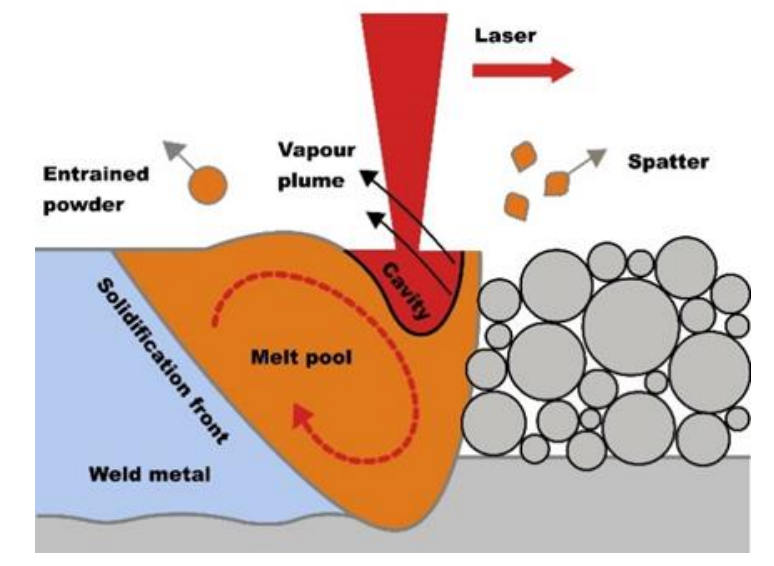

Fig. 4. Efficient processing - optimal combination of speed power [2]

The moving vapour plume creates an environment around the melt pool that is analogous to a weather system. It can entrain powder from next to the weld track, drawing it towards the laser beam through the Bernoulli effect and then ejecting it outwards. Some of this material will be heated as it passes through the laser, while other material is blown around by the induced gas flow in the form of 'winds' adjacent to the laser beam.

\subsection{Lack of fusion case}

If we use less power for a given speed, then the melt pool will be smaller. This means that it is likely to experience less turbulence and generate less spatter as it solidifies more rapidly. The vapour plume will also be less vigorous and so entrainment of neighbouring powder will also be reduced. Unfortunately, the lower laser energy may not penetrate deeply enough to fully melt the powder layer and the top surface of the solid metal below. This leaves unmelted powder beneath the melt pool, resulting in excessive porosity and risk of delamination, as clarified in [2].

\subsection{Keyhole formation}

When too much power is used for a given speed, we see excess penetration of the laser into the metal under the layer of powder, forming a so-called keyhole (Fig. 5). This deep melt cavity in the surface sees metal vapour being ejected more vertically than before. Internal reflections of the laser energy within the cavity trap more heat deeper in the material, leading to a deeper, longer-lasting melt pool. This increase in energy input will increase melt pool turbulence and spatter formation, whilst a stronger 'weather system' will lead to more powder entrainment.

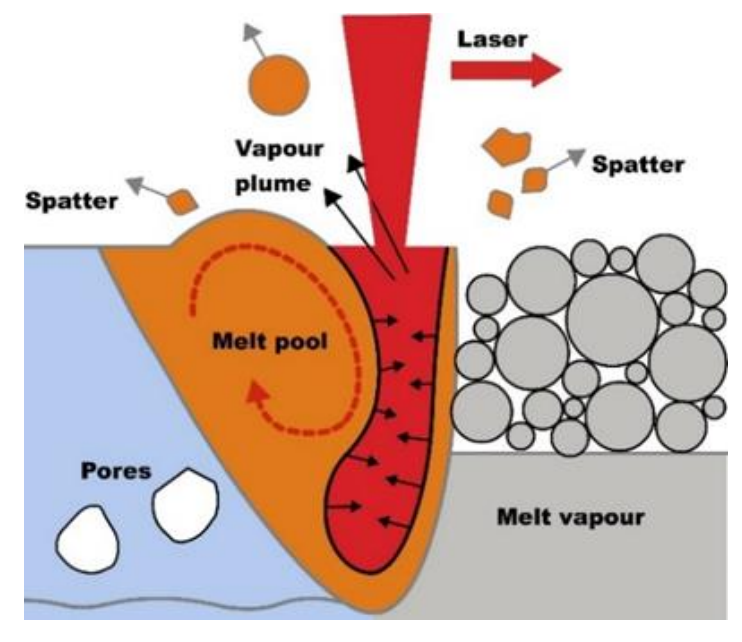

Fig. 5. Excessive keyhole effect [2] 
Where the keyhole becomes unstable, the melt pool can collapse in on the cavity to form a pore of inert gas at the base. Such pores may not close up as the melt pool solidifies, generating sub-surface porosity in the welded metal. A greater degree of re-melting of the layers below will also occur, affecting the microstructure of the solidified material (Fig. 5). At even higher speeds, the melt pool can become unstable. High surface tension gradients can lead to the formation of voids behind the laser beam that expand as the laser moves on, causing the melt pool to break apart into separate islands that solidify as beads [2].

\subsection{Balling up effect}

As is shown in Fig. 3, it is possible to increase both power and speed together to build faster using values from the processing window [3]. However, unfortunately, only to a certain extent. Higher powers and speeds stimulate a tendency of the molten metal to form disconnected metal balls, rather than a flat molten region on the powder bed surface. This phenomenon called 'balling up' is caused by surface energy driving force that limits the surface area to volume ratio, which is minimized as a sphere. Therefore, when surface tension forces overcome a combination of dynamic fluid, gravitational and adhesion forces, the molten metal forms a ball [1]. This phenomenon is undesirable, as are keyhole formations or insufficiently sintered parts, which cause deterioration of surface quality and mechanical properties.

\subsection{Melt pool size}

Process parameter choices determine melt pool size. Their impact is visible in Fig. 6, which comes from high speed thermographic measurements of a single track laser scan [2]. Measuring an Inconel melt pool, it was observed that the melt pool length is roughly constant for different scan speeds at the same laser power. However, the melt pool width, and hence area, increases as the speed reduces. In this case, at $200 \mathrm{~W}$ laser power, the length of the melt pool is approx. 0.6 $\mathrm{mm}$ at speeds varying from $200 \mathrm{~mm} / \mathrm{s}$ to $800 \mathrm{~mm} / \mathrm{s}$. The wider (and thus deeper) melt pool created at slower scan speeds contains more thermal energy and so takes longer to solidify [3].

Figure 6 shows average melt pool length mapped onto the P-W space. The colours of the laser scan tracks (moving from right to left) indicate measured temperatures (in detectable range) from $550^{\circ} \mathrm{C}$ (red) to $1038^{\circ} \mathrm{C}$ (white-yellow), the shaded region in the upper left corner indicates where keyholing is expected.

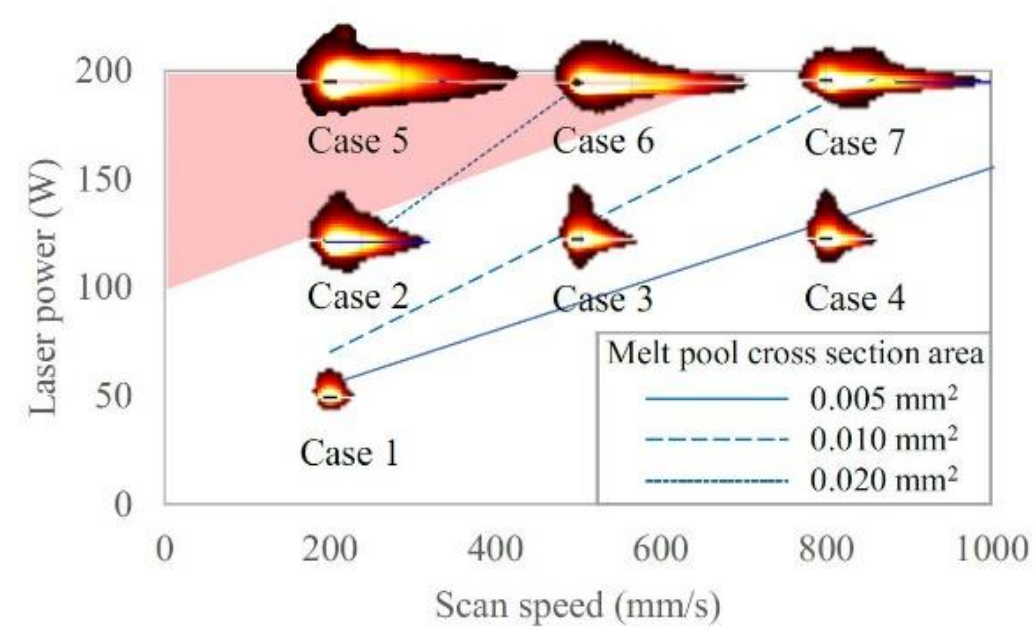

Fig. 6. Infrared images of melt pools mapped onto the Laser power - Scan speed space [3]

\section{The setting of the experiment}

To verify the above mechanism and find a field of efficient combinations of chosen laser power and scan speed values, an experiment was prepared and conducted using the EOS M290 machine. Experimental samples were printed with individually set process parameters, which were processed using EOS Print 1.6 software. In contrast to the single layer and single track scanning experiments (described e.g. in [7]), this experiment was performed with a special effort to ensure real printing conditions. The experimental samples had the form of cubes with dimensions 10x10x10 mm, where the lower half of the cube was a support structure with a cone support in the middle. See Fig. 2.

The settings of this experiment are based on a database of default and exposure settings specified for different kinds of powder, different layer types and layer thicknesses. The laser power values and scan speeds from this database were used in a matrix containing combinations of 11 rows and 11 columns. Thus, these combinations did not only include already used values (or values near to them), but also practically useless values, which would mean too high or too low energy input. 
Therefore, the energy density $\left(E_{D}\right)$ delivered by the laser beam was calculated for all of these combinations and an interval of effective values was determined according to combinations of proven settings. In contrast to significantly lower values of energy density, values that significantly exceed this interval were omitted, because their possible heat-induced deformation could cause a collision with the recoater. The rest of the samples with lesser probability of collision were separated from the other samples so as not to endanger their successful sintering. All samples were aligned in 11 mutually shifted rows and columns with $1 \mathrm{~cm}$ spacing and 5-degree rotation (for smoother printing), as shown in Figure 8.

An AM machine of type EOS M 290 was used for this experiment. This model has a building volume $250 \times 250 \times$ $325 \mathrm{~mm}$ and uses a $\mathrm{Yb}$ fibre laser with max. $400 \mathrm{~W}$ beam power, up to $7.0 \mathrm{~m} / \mathrm{s}$ scan speed and $80 \mu \mathrm{m}$ focus diameter. EOS Maraging Steel MS1 (AKA 1.2709 or X3NiCoMoTi 18-9-5) with $8.0 \mathrm{~g} / \mathrm{cm}^{3}$ density was used as build material, and Argon as the protective atmosphere.

The following parameters were used for the experiment:

Core Exposure type:

- Hatch distance: $0,1 \mathrm{~mm}$

- Scan speed: 200, 300, 450, 600, 800, 1000, 1500, 2000, 2400, 2800, $3200 \mathrm{~mm} / \mathrm{s}$

- Laser power: 20, 35, 50, 80, 100, 138, 150, 195, 250, 305, $350 \mathrm{~W}$

- Beam offset: $0 \mathrm{~mm}$

- Hatch stripe width: $10 \mathrm{~mm}$

- Hatch stripe overlap: 0,08 mm

- With selected X, Y Hatching, Skywriting, Offset, Alternating and Rotated

- Upskin and Downskin - Thickness: 0

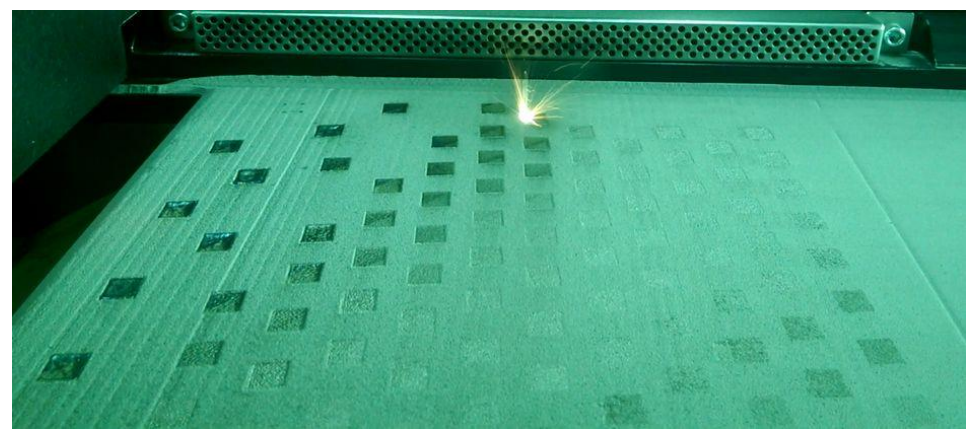

Fig. 7. Experimental samples (cubes) during the sintering process

\section{Results and evaluation of the experiment}

A sintering process of 118 experimental units/cubes took 9.25 hours. Ten units with higher $\mathrm{E}_{\mathrm{D}}$ began to cause vibrations of the recoater during new layer depositions. Subsequently, their sintering was interrupted because of the increased risk of imminent collision with the recoater. Thirty units with low value of $E_{D}$ were not sintered at all or were sintered only partially. These units, located in the front and on the right part of Figs. 8 and 11, did not receive a sufficient amount of energy and therefore were not successfully sintered - these units fell apart leaving their support structures (sintered with default parameters) on the platform.

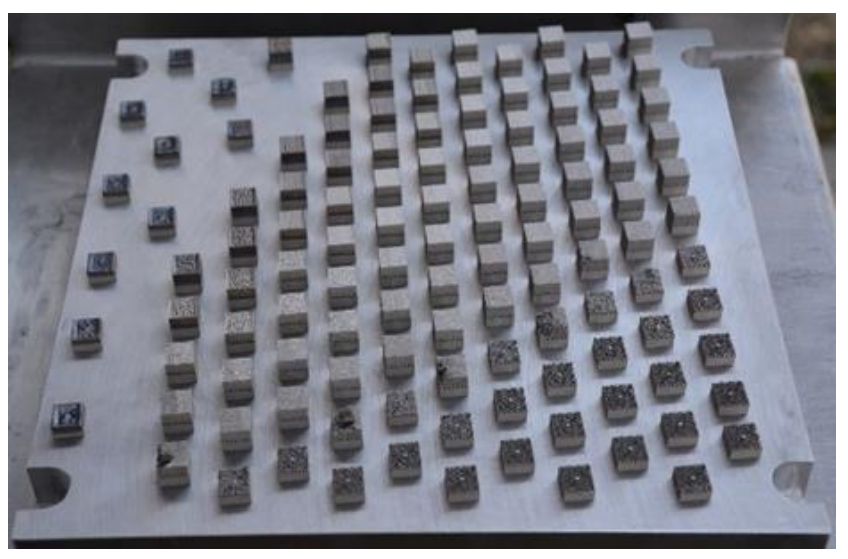

Fig. 8. Result of the experiment - all samples on the printing platform 
In the next phase, after cleaning the platform and removing residual powder, all successfully sintered samples were investigated and scanned using an Alicona IFM G4 high-resolution optical 3D microscope. The entire volume of all the samples was sintered using only exposure type Core (Upskin and Downskin type were disabled) so it was possible to observe the real (i.e. uncorrected finishing combinations of parameters, such as e.g. Upskin type) effects of the selected process parameters and to see individual laser tracks. The Alicona microscope scanned, measured surfaces of samples and created a 3D model of every sample. These 3D models enabled thorough exploration and evaluation of the sample surfaces that show signs of all the above-mentioned phenomena. Figure 9, which contains selected snapshots of 3D models and of enlarged surfaces, shows satisfactorily flat surfaces - the result of suitable combinations of parameters. In contrast to them, surfaces with visible balling up effect, keyhole and spatter formations are included in Fig. 10. For comparison, there is shown (in Fig. 9) a snapshot of a comparable sample printed with the default parameters designed for sintering surfaces. This Upskin exposure type uses a combination of laser power and scan speed with values 153 - 600, which creates quite a smooth surface.

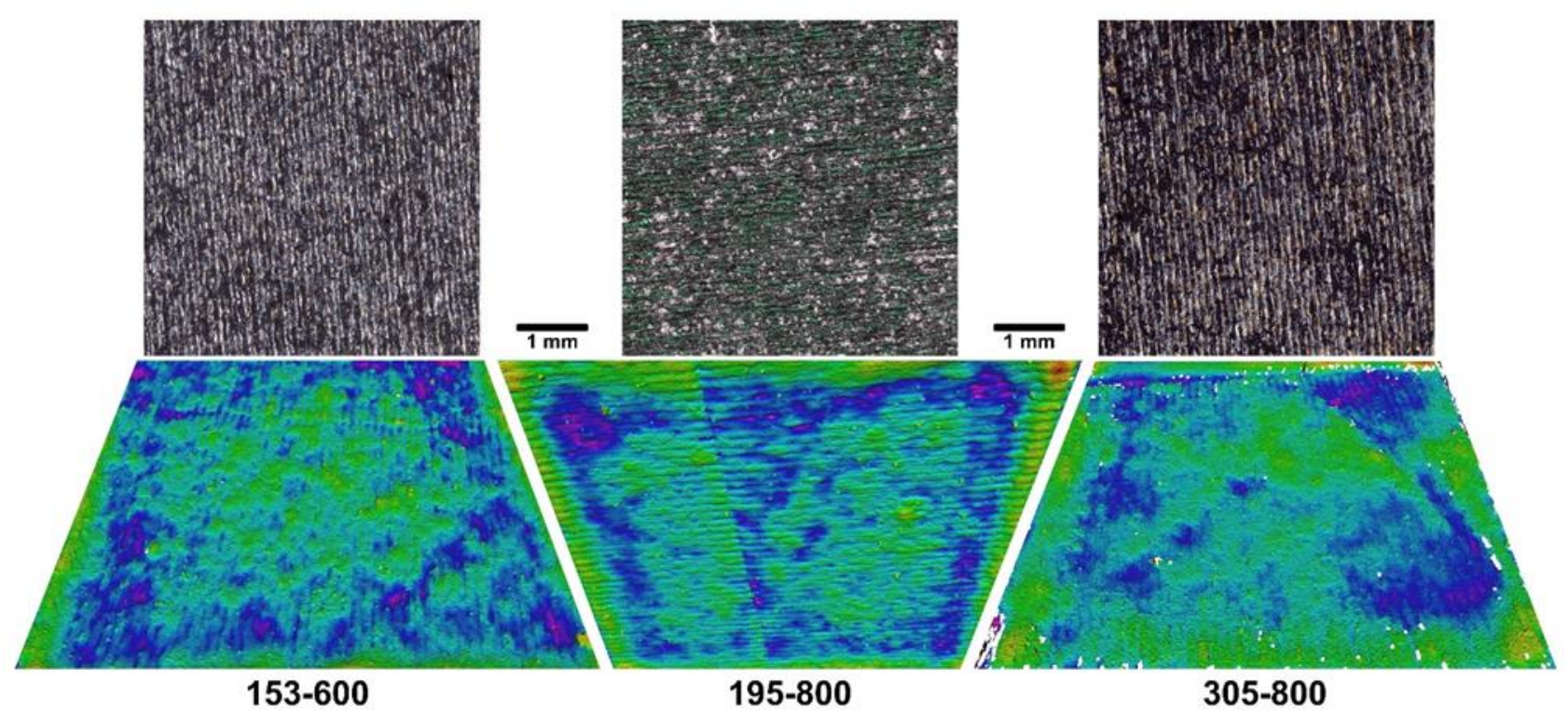

Fig. 9. Pictures of 3D models and enlarged surface cut-outs of selected samples (labelled by P-V combination)

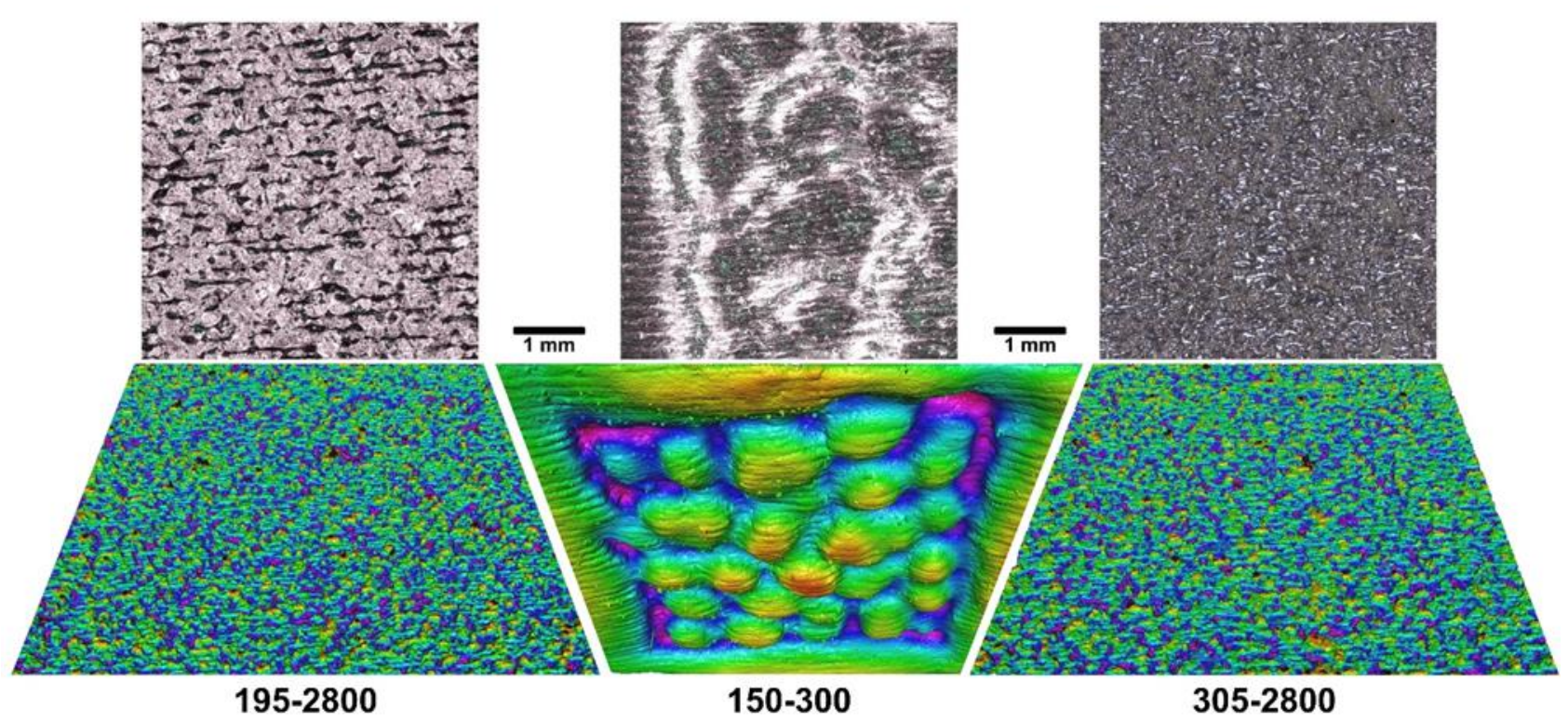

Fig. 10. Pictures of 3D models and enlarged surface cut-outs of selected samples with inferior surface

The matrix below (Fig. 11) contains an overview of the printing platform with all experimental samples. Every sample represents a combination of laser power ( $\mathrm{P}$ value in rows) and scan speed ( $\mathrm{V}$ value in columns) and shows the values of given energy densities. Samples (squares) with bold boundaries are shown in detail in Figs. 9 and 10. All samples in the matrix are marked by type and evaluation in the following way: 
- Crossed out squares - unsintered samples as a result of lack of fusion

- Dark squares with crossed out values - omitted samples because of too high energy density level

- Dark squares - overheated samples, whose printing was interrupted

- Hatched squares - samples with keyhole formations

- Dotted squares - samples with visible balling up effect and spatter formations

- White squares - samples with satisfactory quality, the area of operating window

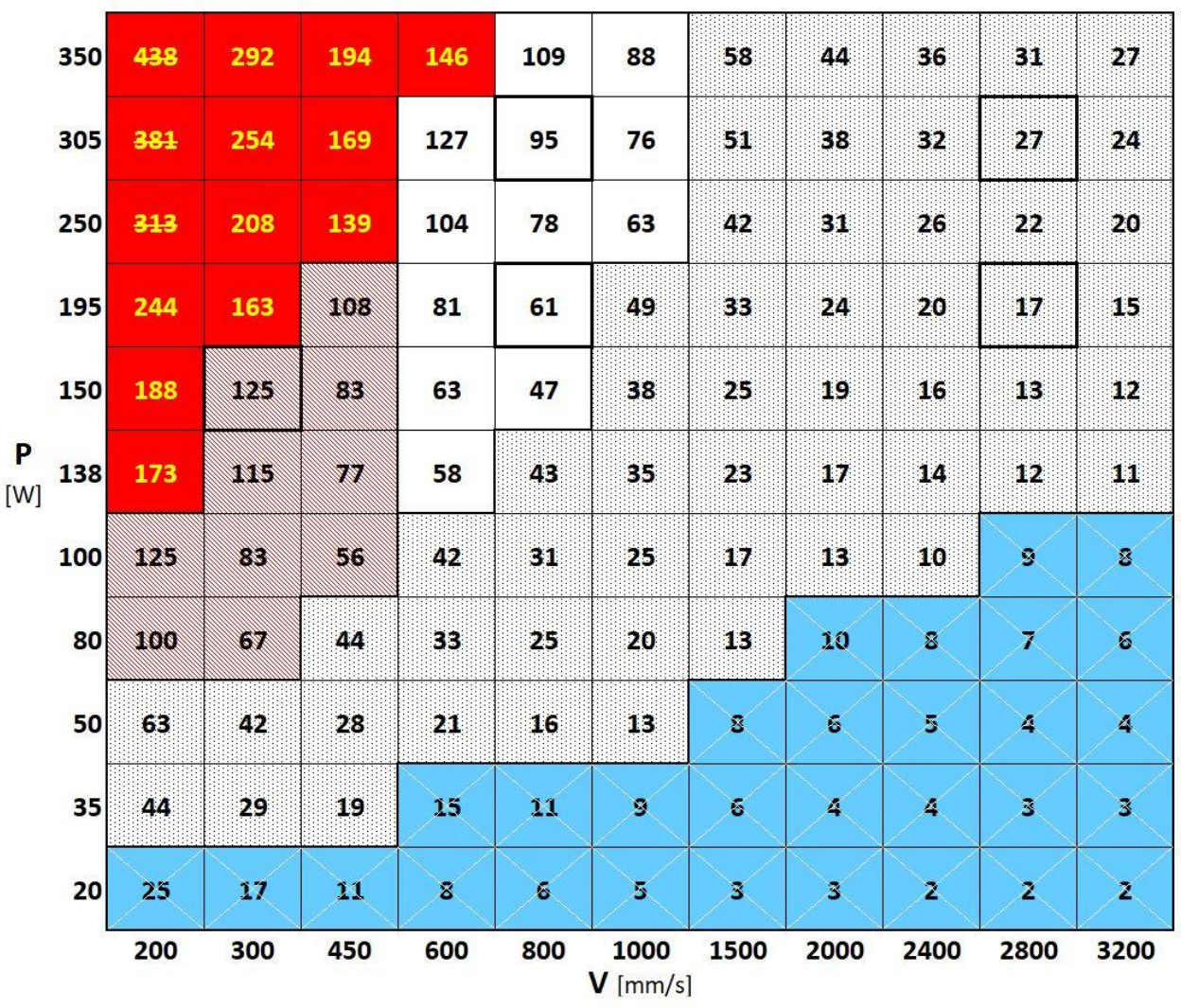

Fig. 11. Matrix of all laser powers and speeds combinations with values of energy densities and labelling of samples

As can be seen in the matrix above, the operation window includes combinations of very different values, which could be used as possible process parameters for sintering volume type parts. Some of the discovered combinations have almost the same or even better results than the ones sintered using default parameters. For instance, units with values 305-800 or 195-800 have similar features too Upskin parameters with values 153 - 600 (see Fig. 9), but with $800 \mathrm{~mm} / \mathrm{s}$ the sintering process would be faster. And the printing time is very important, because machine costs represent about $80 \%$ of total production costs [6]. However, in this context, it is necessary to add that the Upskin exposure setting is used only for the upper surface and the major parts of (not only) bulky pieces are sintered using the Core exposure type. This type uses a combination of values 285-960, which creates not so smooth layers as Upskin type, but creates them faster. The value of $960 \mathrm{~mm} / \mathrm{s}$ lies almost on the border of the processing window so, in this case, room for further acceleration of the sintering process is very limited. Nonetheless, the operating window allows the selection of parameter combinations as needed (e.g. possible ways to decrease the energy input in the case of parts with difficult laser heat dissipation) and specifies the area in which it is possible to find better combinations of laser power and scan speed.

\section{Conclusion}

This article attempts to describe the mechanism of powder bed fusion and the processes that take place inside it during laser sintering of metal powder. Certain combinations of laser power and scan speed were tested in the experiment conducted in real conditions using an EOS M290 machine. Each experimental sample (cube) was printed with a different combination of laser power and scan speed, and the shape and surface quality of successfully sintered units were evaluated using Alicona optical 3D microscope.

Processes accompanying powder bed fusion were explained using the theory described, which quite accurately predicts the observed phenomena and structures that are achieved during the experiment. As verified and found, if the laser beam moves too fast with too little power, then not fully melted areas will appear with increased porosity and surface roughness (e.g. samples with low $\mathrm{E}_{\mathrm{D}}$ in the front and right front corner in Fig. 8). 
On the other hand, if too much power for the given speed is applied, then the melt pool could be overheated, which could cause deeper energy penetration and lead to an undesired effect called 'keyhole formation' (e.g. sample 150-300 in Fig. 10). Between these two extremes is located an operating window where it is possible to achieve satisfactory part density (see Fig. 9). In this operating window, the laser energy is sufficient to fully melt the powder and the underlying metal without penetrating too deeply. Appropriate values of laser power and scan speed could be increased together to sinter faster, but only to a certain extent. There is a limit to how hard and fast it is possible to go, beyond which the melt pool behaviour becomes unstable and an effect known as 'balling up' appears (e.g. sample 195-3200 in Fig. 10). At higher energy input it is also possible to observe the increasing presence of spatter formation, which represents disrupted laser tracks (e.g. sample 305-2800 in Fig. 10) and therefore impaired quality of individual sintered layers and degraded mechanical properties of the final part.

This experiment confirms some assumptions about the powder bed fusion mechanism, discovered a broad map of a processing window and several favourable combinations of processing parameters. However, this is just the first step in the search for particular settings. The next step is to conduct an experiment, which would take place only in the processing window close to promising values. Experimental samples obtained in this way would be subjected to mechanical testing and its structure analysed using SEM to discover exactly the best settings. Subsequent research would also be focused on the impact of the hatching speed at constant energy density, the impact of heat accumulation on the powder bed fusion mechanism and on the final properties of sintered parts with different structures, and also the influence of other process parameters (e.g. spot size, scanning strategies and spacing) on the whole process of sintering.

\section{Acknowledgments}

The article has been prepared under project LO1502 'Development of the Regional Technological Institute' under the auspices of the National Sustainability Programme I of the Ministry of Education of the Czech Republic aimed at supporting research, experimental development and innovation.

\section{References}

[1] Ian Gibson, I.; Rosen, D.; Stucker, B.; (2015). Additive Manufacturing Technologies, Springer-Verlag New York, ISBN 978-1-4419-1119-3, New York, USA

[2] Saunders, M. (2018). How process parameters drive successful metal AM part production, Available from: http://issuu.com/inovar-communications/docs/mam_summer_2018_sp?e=32443561/63203285 Accessed: 201808-16

[3] Heigel, J.C.; Lane, B.M. (2017). Measurement of the melt pool length during single scan tracks in a commercial laser powder bed fusion process, Available from: https://www.nist.gov/publications/measurement-melt-pool-lengthduring-single-scan-tracks-commercial-laser-powder-bed Accessed: 2018-08-24

[4] Calignano, F.; Manfredi, D.; Ambrosio, E.P.; Iuliano L.; Fino, P. (2013). Influence of process parameters on surface roughness of aluminum parts produced by DMLS, The International Journal of Advanced Manufacturing Technology, Volume 67, Issue 9-12, August 2013, pp. 2743-2751, ISSN 0268-3768

[5] Williams, J.; Miller, D.; Deckard, C. (1996) Selective Laser Sintering Part Strength as a Function of Andrew Number, Scan Rate and Spot Size, Available from: http://sffsymposium.engr.utexas.edu/Manuscripts/1996/199663-Williams.pdf Accessed: 2018-09-10

[6] Nozar, M; Zetková, I, Hanzl, P \& Dana, M (2017). A Customer's View on Key Aspects of Metal Additive Manufacturing, Proceedings of the 28th DAAAM International Symposium, pp.957-966, B. Katalinic (Ed.), Published by DAAAM International, ISBN 978-3-902734-11-2, ISSN 1726-9679, Vienna, Austria DOI: 10.2507/28th.daaam.proceedings. 133

[7] Hanzl, P.; Zetek, M.; Bakša, T.; Kroupa, T. (2015). The Influence of Processing Parameters on the Mechanical Properties of SLM Parts, Available from: http://www.daaam.info/Downloads/Pdfs/proceedings/proceedings_2014/ 176.pdf Accessed: 2017-06-15

[8] EOSPRINT 1.6 - Operating instructions (2017). EOS GmbH - Electro Optical Systems, Edition 04.17, pp. 114-124, München 2017

[9] Alexander, P.; Allen, S.; Dutta, D. (1998). Part orientation and build cost determination in layered manufacturing. Available from: http://www.sciencedirect.com/science/article/pii/S0010448597000833, Accessed: 2017-09-01 